\title{
Multiplex Amplicon Quantification (MAQ), a fast and efficient method for the simultaneous detection of copy number alterations in neuroblastoma
}

\author{
Candy Kumps' ${ }^{1}$ Nadine Van Roy', Lien Heyrman2,3, Dirk Goossens²,3, Jurgen Del-Favero2,3, Rosa Noguera4, \\ Jo Vandesompele1, Frank Speleman ${ }^{1}$ and Katleen De Preter*1
}

\begin{abstract} detect the important genomic aberrations.

\section{Background}

Recurrent alterations in DNA copy number are a common feature in many cancers and typically target biological pathways and processes that contribute to cancer pathogenesis. The diagnostic and prognostic relevance of these chromosomal imbalances (gains, losses and amplifications) has been demonstrated in an increasing number of tumor entities [1]. For the detection of unbalanced chromosomal aberrations different profiling platforms are commercially available. Array CGH is a commonly applied high-resolution genome-wide screening method for research and diagnostic purposes [2] but currently remains relatively expensive and labor-intensive. Alternatively, PCR-based methods for the simultaneous detection of copy number changes in selected chromosomal regions such as Multiplex Ligation-dependent Probe
\end{abstract}

Background: Cancer genomes display characteristic patterns of chromosomal imbalances, often with diagnostic and prognostic relevance. Therefore assays for genome-wide copy number screening and simultaneous detection of copy number alterations in specific chromosomal regions are of increasing importance in the diagnostic work-up of tumors.

Results: We tested the performance of Multiplex Amplicon Quantification, a newly developed low-cost, closed-tube and high-throughput PCR-based technique for detection of copy number alterations in regions with prognostic relevance for neuroblastoma. Comparison with array CGH and the established Multiplex Ligation-dependent Probe Amplification method on 52 neuroblastoma tumors showed that Multiplex Amplicon Quantification can reliably

Conclusion: Multiplex Amplicon Quantification is a low-cost and high-throughput PCR-based technique that can reliably detect copy number alterations in regions with prognostic relevance for neuroblastoma.

* Correspondence: Katleen.Depreter@Ugent.be

1 Center for Medical Genetics, Ghent University Hospital, Ghent, Belgium Full list of author information is available at the end of the article
Amplification (MLPA) [3] are well established and suited to be applied on a routine basis [4].

In this study, we evaluated the performance of a new method for relative quantification of specific DNA sequences in routine laboratory practice, called Multiplex Amplicon Quantification (MAQ) [5-9]. For this purpose, a MAQ assay was specifically designed for neuroblastoma (NB), the most common solid extra-cranial pediatric malignancy [10]. This is a good model for MAQ validation as intensive research of genomic imbalances has revealed insights into the clinical and biological heterogeneity of this tumor [11]. More specifically, prognostic relevant, critical regions of loss and gain in NB have been delineated, the most important being $1 \mathrm{p}$ deletion, $\mathrm{MYCN}$ amplification, $3 p$ deletion, $11 q$ deletion and $17 q$ gain [1216]. Of further importance, new therapeutic protocols based on the presence or absence of these segmental abnormalities are in progress [16]. The MAQ-NB assay allows the detection of CNAs in the prognostic relevant regions in NB. In this study, MAQ results were compared 
to copy number changes detected with MLPA on a series of $52 \mathrm{NB}$ for which detailed array CGH profiles were available.

\section{Methods}

Neuroblastoma tumor samples and cell lines

A representative series of NB tumor samples was collected prior to therapy (34 cases from the Ghent University Hospital, Belgium and 14 from the Medical School of Valencia, Spain) (see Additional File 1). Of these $48 \mathrm{NB}$ samples, 17 samples present with only numerical and 31 with segmental aberrations (sometimes in a numerical background). MYCN amplification occurs in 19 cases. Additionally, $4 \mathrm{NB}$ cell lines with segmental aberrations of which 3 with $M Y C N$ amplification were included in this study (NB-19, GI-CI-N, SJ-NB-10, LAN-6). DNA was isolated from NB tumors, cell lines and control samples using the Qiagen DNA isolation kit (QIAgen) according to the manufacturer's instructions.

\section{Multiplex Ligation Probe Amplification (MLPA)} For MLPA analysis, the SALSA NB kits P251 (targeting chromosome 1, 3 and 11) and P252 (targeting chromosome 2 and 17) (MRC-Holland) were used to investigate DNA aberrations in specific chromosome regions of interest for NB [17]. The third NB kit P253, targeting chromosome 4, 7, 9, 12 and 14, was not used for this study. In the case of $M Y C N$ amplified NB samples, a $M Y C N$ silencing solution was added. Target regions are indicated in Figure 1. $100 \mathrm{ng}$ of tumor DNA was used for each MLPA reaction. In each experiment three control samples were analysed, allowing accurate normalisation, including an EBV cell line, constitutional blood from a healthy individual and human genomic DNA (Roche). MLPA was performed according to the manufacturer's protocol with minor adaptations in the PCR step. Briefly, PCR was performed using $10 \mu \mathrm{l}$ of the ligase-product and $40 \mu \mathrm{l}$ of PCR-mix containing $2 \mu \mathrm{l}$ of SALSA primers, $2 \mu \mathrm{l}$ SALSA enzyme dilution buffer, $4 \mu$ l SALSA PCR buffer and $0.5 \mu \mathrm{l}$ polymerase, diluted in HPLC water, prepared on ice.

\section{Multiplex Amplicon Quantification (MAQ)}

For MAQ analysis, three kits were designed, MAQ1, MAQ2 and MAQ3 as described in the results section. 50 ng of tumor DNA was needed for each reaction of the MAQ analysis. For accurate normalisation, two control samples were included in each experiment, i.e. constitutional DNA from a healthy individual and human genomic DNA (Roche). After addition of $10 \mu \mathrm{l}$ of PCR mix and $0.075 \mu$ of Taq polymerase, samples were heated in a thermocycler with a heated lid (PTC200, Bio-Rad) for 2 minutes at $98^{\circ} \mathrm{C}$ for activation of the polymerase. Further PCR conditions required 22 cycles, including dena- turation at $95^{\circ} \mathrm{C}$ for 45 seconds, annealing at $60^{\circ} \mathrm{C}$ for 45 seconds and extension for 2 minutes at $68^{\circ} \mathrm{C}$. This was followed by a final extension step at $72^{\circ} \mathrm{C}$ for 10 minutes.

\section{Capillary electrophoresis and data analysis}

For both MLPA and MAQ, resulting PCR products were analysed by capillary electrophoresis. A mixture of $8.5 \mu \mathrm{l}$ formamide (HiDi, Applied Biosystems) and $0.5 \mu$ internal lane standard (Rox500, Applied Biosystems) was prepared and $1 \mu \mathrm{l}$ of the PCR-product was added. After a 2 minute denaturation-step at $95^{\circ} \mathrm{C}$, the samples were analyzed on the ABI3130XL (Applied Biosystems) capillary electrophoresis system. The raw data generated by fragment analysis were analysed in a specially designed software program Coffalyser [18] and MAQ-S [5] for MLPA and MAQ, respectively. Both programs were designed to calculate and visualise the normalised peak area or dosage quotient which reflects copy number of each target amplicon. Dosage Quotients (DQ) are calculated using the reference amplicons (in regions with low occurrence of aberrations) and the control samples (without genomic aberrations). For one reference amplicon (RefA) and one control sample (ctr), the DQ of a target amplicon (TarA) in sample (s) is as follows: DQ $=[\operatorname{Tar} A(s) / \operatorname{RefA}(s)] /$ $[\operatorname{Tar} A(\mathrm{ctr}) / \operatorname{RefA}(\mathrm{ctr})]$. Based on the data of all reference amplicons and control samples, a final DQ is calculated as the mean of the individual DQs and the standard deviation of the DQs (visualized as error bars) is a measure of stability.

\section{Scoring rules for MLPA and MAQ}

Optimal scoring thresholds were applied for MLPA and MAQ. For MLPA scoring (linear scale) we applied the following criteria. Regions were scored as segmental deletions or gains if the dosage quotients of at least 2 consecutive loci is 0.25 below or above 1 , respectively [19] or when more than $75 \%$ of the probes show a decrease or increase of at least 0.15 , respectively. When no segmental aberrations were detected in the tumor, the presence of numerical changes was evaluated. Regions are scored as whole chromosomal losses or gains if at least $75 \%$ of the dosage quotients of both $p$ and $q$ arm have a value below or above 1 , respectively and more than half of the dosage quotients show an decrease or a increase of at least 0.1. MYCN amplification is scored if the 2 dosage quotients for the MYCN locus increase with at least 3. For MAQ scoring (linear scale) we applied the following criteria. Regions were scored as segmental deletions or gains if the dosage quotients of at least 2 consecutive loci is 0.2 below or above 1 , respectively (according to the manufacturer's instructions). When no segmental aberrations were detected in the tumor, the presence of numerical changes was evaluated. Regions are scored as whole chromosomal losses or gains if at least $75 \%$ of the 


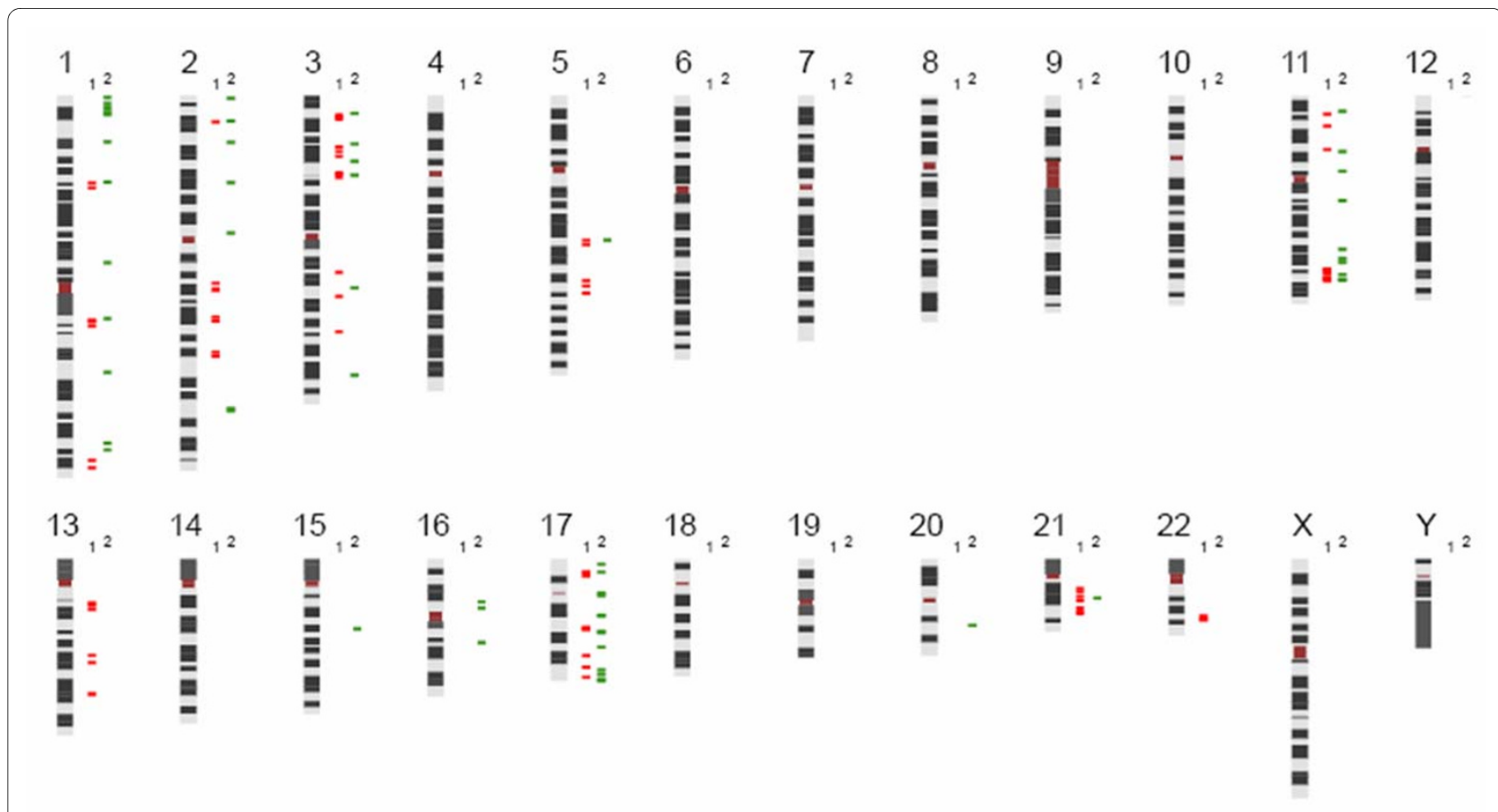

Figure 1 Karyo view indicating regions covered by MAQ (1) and MLPA amplicons (2).

dosage quotients of both $\mathrm{p}$ and $\mathrm{q}$ arm have a value below or above 1 , respectively and more than half of the dosage quotients show an decrease or an increase of at least 0.1 . For scoring of MYCN amplification the 3 dosage quotients for the MYCN locus should increase with at least 3.

\section{Array Comparative Genomic Hybridisation (array CGH)}

Samples were profiled on an in-house developed $1 \mathrm{Mb}$ resolution BAC array as previously described [13] (18 samples) or on a custom designed $44 \mathrm{~K}$ array (Agilent Technologies) (34 samples) enriched for critical regions in NB (e.g. 1p, 2p, 3p, 11q, 17). Utilizing random prime labeling (BioPrime ArrayCGH Genomic Labeling System, Invitrogen), $400 \mathrm{ng}$ of tumor and control DNA (DNA from an EBV cell line or male control DNA, Promega) was labeled with Cy3 and Cy5 dyes (GE healthcare). Further processing was then performed according to the manufacturer's instructions (Agilent Technologies). Slides were scanned using an Agilent scanner (Agilent Technologies), features were extracted using the feature extraction v10.1.1.1 software program and further processed with an in-house developed visualisation software program arrayCGHbase [20,21]. Array CGH profiles were evaluated manually after application of the circular binary segmentation (CBS) algorithm, which is an algorithm to dissect genomic array data into regions of equal copy number by applying a maximal $\mathrm{t}$-statistic with a permutation reference distribution to determine the change- points [22]. Gains are indicated in green (CBS value > $0.3)$; losses are indicated in red (CBS value $<-0.3)$.

\section{Results}

Construction of the MAQ neuroblastoma assay

The MAQ technique consists of the quantification of fluorescently labelled test and control amplicons, obtained by a single multiplex PCR (mPCR) amplification. As a first step in the construction of the MAQ neuroblastoma assay, primers were designed in the critical regions of loss (1p, 3p and 11q) and gain (2p -more specifically the $M Y C N$ locus- and 17q) for NB and their respective opposite chromosome arm $[12,13,15]$. Specialised software [5] was used for the development of PCR primer sets within the chromosome regions of interest with a very high multiplexing degree, targeting up to 40 sequences each with a unique length. In this way, the NB-MAQ assay was created consisting of three kits. MAQ1 contains a total of 21 primer pairs with target regions on chromosome 3 and 17 and MAQ2 contains 19 primer pairs targeting chromosome 1 and 11 . For the detection of $M Y C N(2 \mathrm{p})$ status, a separate kit (MAQ3) was designed, containing 9 primer pairs for chromosome 2 . This was necessary since the high levels of $(M Y C N)$ amplification interfered with reliable analysis of other amplicons generated in the multiplex PCR. Additionally, each kit includes 8 or 9 primer sets, covering chromosomal regions that show the lowest frequency of copy number changes in NB (chromosome $5 q, 13 q, 21 q, 22 q)$ and serve as reference sequences used 
for accurate normalisation. These regions were carefully selected through screening of the chromosomal status on array CGH profiles of $1000 \mathrm{NB}$ cases (De Preter et al., in preparation). The MAQ target regions are indicated in Figure 1. More detailed information on the target regions of the MAQ kits can be found in Additional File 2.

\section{Evaluation of the MAQ assay by comparison with MLPA and array CGH}

The performance of the MAQ assay for the detection of CNAs in prognostic relevant regions was evaluated by comparing results of $48 \mathrm{NB}$ tumor samples and $4 \mathrm{NB}$ cell lines with corresponding MLPA and array CGH profiles (Additional File 3). For this analysis, each sample was screened once and scoring was applied as described in the Methods section. Based on array CGH results (Additional File 4), 2 groups were defined. One group consists of 17 tumor samples which presented exclusively with numerical aberrations (whole chromosome gains and losses), whereas the second group of 35 samples was characterised by the presence of segmental aberrations, sometimes in a numerical background (15/35). Two NB tumor profiles representative for each subgroup are shown in Figure 2, illustrating high concordance between array CGH, MLPA and MAQ results.

Performance of MLPA and MAQ for the detection of aberrations in each of the prognostic relevant regions is summarised in Table 1. The area under the curve (AUC) of the receiver operating curve (ROC) was used as a measure for accuracy, summarizing the sensitivity and specificity for a given test. High-quality tests with almost perfect performance will have an AUC approaching $100 \%$. ROC curves are shown in Additional File 5. Array $\mathrm{CGH}$ was used as golden standard for these analyses. For the subset of tumors with segmental aberrations, performance for detecting 1p, MYCN, 3p, 11q and 17q status was very high for both techniques with average AUC of 97.2\% and 98.9\% for MLPA and MAQ, respectively. The false positive rate for MLPA and MAQ was $4.7 \%$ and $0.9 \%$, respectively. For MLPA, no false negatives were detected while for MAQ one false negative was detected at chromosome $1 \mathrm{p}$ (false negative rate of $1.1 \%$ ). It should be noted that the reference amplicons in this individual were all located in regions with a low level loss, therefore the lost 1 p region appeared as normal.

For the 17 cases presenting with only numerical aberrations, performance was evaluated for whole chromosome changes. Overall, performance rates were less optimal for these tumors with average AUC of $78.5 \%$ and $82.5 \%$ for MLPA and MAQ, respectively. When looking more into detail, the AUC remains high for chromosome 17 for both techniques (AUC is 0.941), demonstrating that high level gains are easily detected. While lower performance is seen for chromosome 1, 2, 3 and 11. As well for array
CGH as for MLPA and MAQ, detection of whole chromosome gains and losses in near-triploid tumors is challenging. Nevertheless, these aberrations can be detected rather unambiguously with array $\mathrm{CGH}$ due to the high amount of oligos used on these platforms (as can be seen in Figure 2B). This problem is inherent to characteristics in NB tumors with numerical aneuploidy where many chromosomes are implicated, including those used as reference for normalisation. No false positives were detected with MAQ and the false positive rate for MLPA was $11.7 \%$, while the false negative rate was $23.5 \%$ and $24.1 \%$, respectively. Importantly, for these near-triploid tumors the only clinically relevant question is whether any segmental aberrations are detected and this is not the case when MAQ is used.

Furthermore, repeatability of MAQ measurements was tested by performing Bland-Altman analysis [23]. From this method the coefficient of repeatability was derived by calculating the average difference \pm 1.96 standard deviation of the difference. MAQ1 measurements of 6 samples ( 4 tumors with segmental aberrations and 2 with numerical aberrations) were found reliably repeatable. The coefficient of repeatability was 0.121 for the tumors with segmental and 0.0987 for the tumors with numerical imbalances and therefore fall within the threshold boundaries used in the scoring procedure.

\section{Discussion}

MAQ is a new PCR-based method that allows to determine the copy number status of multiple loci in a single assay. Similar to MLPA, this technique fills the gap between more expensive genome-wide screening assays and cheaper methods that only provide information on a single locus. We validated MAQ in a model for NB as an alternative for MLPA, using array CGH data as a reference point. This study shows that MAQ is a robust and repeatable method for detection of prognostic relevant CNAs in recurrently affected regions in NB tumors and cell lines. For all investigated samples, $M Y C N$ amplification status, which is one of the strongest prognostic parameters, was accurately determined. In addition, MAQ reliably differentiates between tumors with segmental aberrations (which are correlated to poor prognosis) and tumors with numerical aberrations (correlated to good prognosis) [15]. For the detection of segmental gains and losses of one or more copies in 35 NB samples, performance was very high even when measured in a numerical background (Table 1). Of notice, the few discrepancies measured (both for MLPA and MAQ) could represent very small aberrations, which we anticipate to be very rare. The validity of such putative small aberrations might be examined by MLPA or MAQ assays enriched for probes in this region, ultra-high resolution arrays with dense coverage in this region or even next 


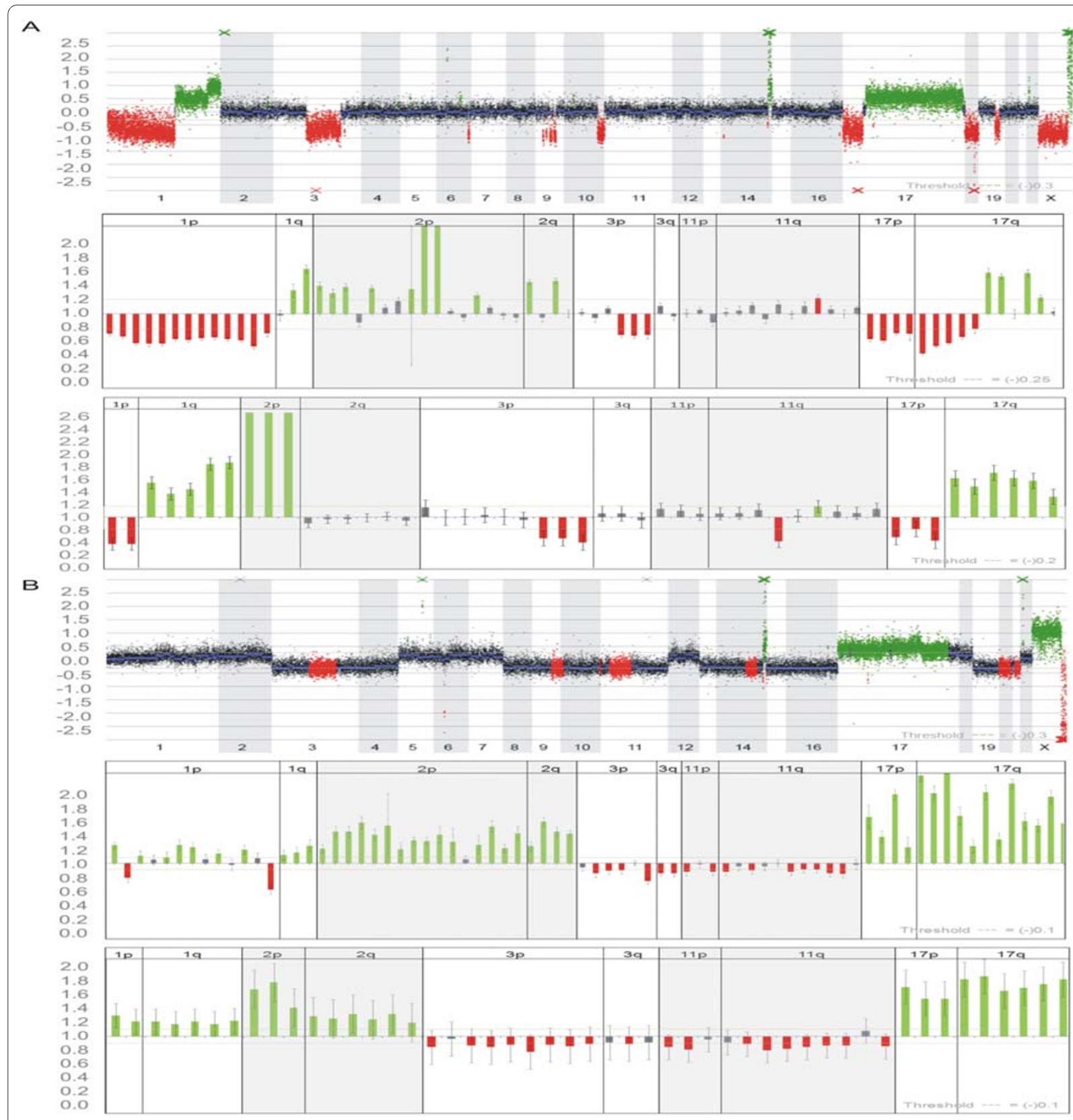

Figure 2 Two representative examples of aCGH, MLPA and MAQ NB tumor profiles A. NB cell line SJNB-10 with segmental aberrations, including $1 p$ deletion, MYCN amplification, 3p deletion, 17q gain. B. NB tumor with numerical aberrations including whole chromosome 1, 2 and 17 gain, and whole chromosome 3 and 11 loss. Gray = normal; Red = loss; Green = gain (according to scoring thresholds). Horizontal bars in array profiles indicate CBS values. Red and green cross marks point at CBS values above or below 0.3 or -0.3 , respectively. In Figure $2 B$ all crosses indicate known copy number variations. Error bars of each dosage quotient (DQ) are based on the standard deviation after summarizing the DQ values using different reference amplicons and 3 (MLPA) or 2 (MAQ) control samples.

generation sequencing but this lies beyond the scope of our study. Detection of whole chromosome imbalances in near-triploid tumors is more challenging for both array CGH and MLPA and MAQ. In general the presence of multiple gains and losses in these tumors hampers normalisation [24]. In our study, 16 out of 17 tumors with numerical aberrations (and even in 15/35 with segmen- tal), one or more reference chromosomes show CNAs, thus further complicating accurate normalisation for MAQ. In the case of NB, the pattern of these tumors is clearly recognisable [13]. In addition, for prognostic purposes, it is not of importance to know which chromosomes are numerically aberrant but whether a distinction can be made between tumors with segmental and numer- 
Table 1: Performance of MLPA and MAQ

\begin{tabular}{|c|c|c|c|c|c|c|c|c|c|c|}
\hline \multirow[b]{2}{*}{ Segmental $(n=35)$} & \multicolumn{2}{|c|}{ Chromosome 1p } & \multicolumn{2}{|c|}{ MYCN status } & \multicolumn{2}{|c|}{ Chromosome 3p } & \multicolumn{2}{|c|}{ Chromosome 11q } & \multicolumn{2}{|c|}{ Chromosome 17q } \\
\hline & MLPA & MAQ & MLPA & MAQ & MLPA & MAQ & MLPA & MAQ & MLPA & MAQ \\
\hline AUC & $94.4 \%$ & $97.1 \%$ & $100.0 \%$ & $100.0 \%$ & $94.3 \%$ & $100.0 \%$ & $97.1 \%$ & $97.1 \%$ & $100.0 \%$ & $100.0 \%$ \\
\hline Sensitivity & $100.0 \%$ & $94.4 \%$ & $100.0 \%$ & $100.0 \%$ & $100.0 \%$ & $100.0 \%$ & $100.0 \%$ & $100.0 \%$ & $100.0 \%$ & $100.0 \%$ \\
\hline Specificity & $88.9 \%$ & $100.0 \%$ & $100.0 \%$ & $100.0 \%$ & $92.0 \%$ & $100.0 \%$ & $95.5 \%$ & $95.5 \%$ & $100.0 \%$ & $100.0 \%$ \\
\hline PPV & $90.0 \%$ & $100.0 \%$ & $100.0 \%$ & $100.0 \%$ & $83.3 \%$ & $100.0 \%$ & $92.9 \%$ & $92.9 \%$ & $100.0 \%$ & $100.0 \%$ \\
\hline \multirow[t]{3}{*}{ NPV } & $100.0 \%$ & $94.4 \%$ & $100.0 \%$ & $100.0 \%$ & $100.0 \%$ & $100.0 \%$ & $100.0 \%$ & $100.0 \%$ & $100.0 \%$ & $100.0 \%$ \\
\hline & \multicolumn{6}{|c|}{ 95\% Confidence Interval AUC(MLPA): 94\%-100\% } & \multicolumn{4}{|c|}{ 95\% Confidence Interval AUC(MAQ): 97\%-100\% } \\
\hline & \multicolumn{2}{|c|}{ Chromosome 1} & \multicolumn{2}{|c|}{ Chromosome 2} & \multicolumn{2}{|c|}{ Chromosome 3} & \multicolumn{2}{|c|}{ Chromosome 11} & \multicolumn{2}{|c|}{ Chromosome 17} \\
\hline Numerical $(n=17)$ & MLPA & MAQ & MLPA & MAQ & MLPA & MAQ & MLPA & MAQ & MLPA & MAQ \\
\hline AUC & $76.5 \%$ & $88.2 \%$ & $92.3 \%$ & $76.9 \%$ & $58.8 \%$ & $76.5 \%$ & $70.6 \%$ & $76.5 \%$ & $94.1 \%$ & $94.1 \%$ \\
\hline Sensitivity & $77.8 \%$ & $77.8 \%$ & $90.0 \%$ & $70.0 \%$ & $46.2 \%$ & $69.2 \%$ & $71.4 \%$ & $71.4 \%$ & $94.1 \%$ & $94.1 \%$ \\
\hline Specificity & $75.0 \%$ & $100.0 \%$ & $100.0 \%$ & $100.0 \%$ & $100.0 \%$ & $100.0 \%$ & $66.7 \%$ & $100.0 \%$ & $100.0 \%$ & $100.0 \%$ \\
\hline PPV & $77.8 \%$ & $100.0 \%$ & $100.0 \%$ & $100.0 \%$ & $100.0 \%$ & $100.0 \%$ & $90.9 \%$ & $100.0 \%$ & $100.0 \%$ & $100.0 \%$ \\
\hline \multirow[t]{2}{*}{ NPV } & $75.0 \%$ & $80.0 \%$ & $75.0 \%$ & $50.0 \%$ & $36.4 \%$ & $50.0 \%$ & $33.3 \%$ & $42.9 \%$ & $100.0 \%$ & $100.0 \%$ \\
\hline & \multicolumn{6}{|c|}{ 95\% Confidence Interval AUC(MLPA): 60\%-97\% } & \multicolumn{4}{|c|}{ 95\% Confidence Interval AUC(MAQ): 72\%-93\% } \\
\hline
\end{tabular}

Performance in tumors with segmental (35) and numerical (17) aberrations for the recurrent NB copy number changes at chromosome 1(p), 2 (MYCN status), 3(p), 11(q) and 17(q). This evaluation includes area under curve (AUC), positive predictive value (PPV) and negative predictive value (NPV).

ical aberrations. Importantly, not a single false positive segmental aberration was detected by MAQ in near-triploid tumors with only numerical aberrations. In addition, whole chromosome 17 gain detection which is one of the typical characteristics of these tumors, was almost perfectly detected, thus allowing assignment of these tumors to the prognostic favorable category.

\section{Conclusion}

We demonstrated that the newly developed MAQ method can be used as a valuable diagnostic tool for reliable detection of copy number changes with prognostic relevance in NB. Overall, PCR-based techniques harbor advantages in comparison to array $\mathrm{CGH}$ such as reduced expenses and sample-handling while maintaining high performance. Moreover the equipment needed, i.e. a thermal cycler and a capillary electrophoresis system, is present in the majority of molecular biology laboratories performing routine diagnostics. As MAQ allows to measure copy number status of as much as 40 targets in one reaction, this is sufficient for many routine tests for which only a well defined and specific number of imbalances needs to be analysed. When comparing MLPA to MAQ, the latter method may have certain advantages (see Table 2). It requires less DNA, reduced handling, experiment time and costs, while it occurs in a single closed-tube reaction, maximally limiting contamination problems. Moreover, this test appears slightly more robust in our hands. At present, guidelines for the diagnostic work-up of NB recommend two independent molecular methods for the assessment of genetic alterations in NB [16]. In this context MAQ can be very well suited as complementary method to array CGH or FISH.

\section{List of abbreviations}

(AUC): Area under the curve; (CBS): Circulary Binary Segmentation; (MAQ): Multiplex Amplicon Quantification; (MLPA): Multiplex ligation-dependent probe amplification; (mPCR): Multiplex PCR; (NPV): Negative predictive value; (NB): Neuroblastoma; (PPV): Positive predictive value; (ROC): Receiver Operating Characteristic. 
Table 2: Overview of parameters important for aCGH, MLPA and MAQ

\begin{tabular}{|c|c|c|c|}
\hline & Array CGH (44 K) & MLPA & MAQ \\
\hline $\begin{array}{c}\text { Number of sequences } \\
\text { investigated per } \\
\text { experiment }\end{array}$ & 44000 DNA sequences & $\begin{array}{c}\text { up to } 45 \text { DNA sequences ( } 5 \\
\text { control probes) }\end{array}$ & $\begin{array}{c}\text { up to } 40 \text { DNA sequences (9-10 } \\
\text { control amplicons) }\end{array}$ \\
\hline Number of reactions & 1 & 2 & 3 \\
\hline Input DNA & $150-400$ ng DNA/reaction & $100-200$ ng DNA/reaction & 50 ng DNA/reaction \\
\hline Throughput & intermediate & high & very high \\
\hline Experiment time & results within $72 \mathrm{~h}$ & results within $24 \mathrm{~h}$ & results within $6 \mathrm{~h}$ \\
\hline Hands-on time & high & medium & low \\
\hline $\begin{array}{c}\text { Detection of numerical } \\
\text { aberrations }\end{array}$ & + & $+/-$ & $+/-$ \\
\hline Material & oven, array scanner, software & $\begin{array}{l}\text { thermocycler and capillary } \\
\text { electophoresis system }\end{array}$ & $\begin{array}{l}\text { thermocycler and capillary } \\
\text { electophoresis system }\end{array}$ \\
\hline $\begin{array}{c}\text { Consumables* } \\
\text { (patient and reference } \\
\text { samples included) }\end{array}$ & $\begin{array}{c}205 € \\
\text { (1 control sample included) }\end{array}$ & $\begin{array}{c}88 € \\
\left(3 \text { control }^{* *} \text { samples included) }\right.\end{array}$ & $\begin{array}{c}66 € \\
\left(2 \text { control }^{* *} \text { samples included }\right)^{2}\end{array}$ \\
\hline $\begin{array}{l}\text { For the price calculation we } \\
\text { umor (approximately } 20 \text { ne } \\
\text { *The MLPA protocol recom } \\
\text { hanufacturers }\end{array}$ & $\begin{array}{l}\text { ned an experiment in which on } \\
\text { in Belgium every year) } \\
\text { o use at least } 3 \text { control sample }\end{array}$ & $\begin{array}{l}\text { B sample is screened which is r } \\
\text { e } 2 \text { control samples are sufficier }\end{array}$ & $\begin{array}{l}\text { nable as NB is a very infrequent } \\
\text { MAQ according to the }\end{array}$ \\
\hline
\end{tabular}

\section{Additional material}

Additional file $\mathbf{1}$ Patient data on $\mathbf{4 8}$ neuroblastoma tumors. Patient data on 48 neuroblastoma tumors including INSS stage, age at diagnosis, MYCN amplification status and survival status.

Additional file 2 Overview primer pools of MAQ1 (A), MAQ2 (B) and MAQ3 (C). Overview of primer pools used for MAQ1 (A), MAQ2 (B) and MAQ3 (C) including details of chromosomal location

Additional file $\mathbf{3}$ Scoring table of $\mathbf{4 8}$ tumors and $\mathbf{4}$ cell lines with segmental (A) and numerical (B) aberrations. Scoring table of 31 tumors and 4 cell lines with segmental aberrations (A). Thirty-five tumors display with segmental aberrations. Scoring was performed at the regions of interest at 1p, MYCN amplification (MNA) status, 3p, 11q and 17q. Scoring table of 17 tumors with exclusively numerical aberrations (B). Seventeen tumors display with only numerical aberrations. Scoring was performed at the regions of interest at for both chromosome arms for chromosome 1, 2, 3, 11 and 17. Red indicates clear loss, light red indicates a loss where the threshold is barely reached, light green indicates a gain where the threshold is barely reached, green indicates clear gain. Black indicates that there was no data obtained.

Additional file 4 Array CGH results of $\mathbf{4 8}$ NB samples and $\mathbf{4}$ cell lines. Array CGH results of 48 NB samples and 4 cell lines that were profiled on 44 Koligoarray or BAC array
Additional file 5 Overview of ROC curves of MLPA and MAQ assays versus array $\mathrm{CGH}$. Overview of ROC curves of MLPA and MAQ assays versus array CGH at 1p, 2p, 3p, 11q and 17q for tumors with segmental and numerical aberrations.

Authors' contributions

CK carried out the experimental work and data analysis, for the array, MLPA and MAQ experiments. NVR participated in the design of the study and helped to draft the manuscript. LH, DG and JDF were involved in the design of the MAQ method. KDP provided the NB regions of interest necessary for the development of the MAQ NB assay. JVDS, FS and KDP conceived of the study and participated in its design and coordination and helped to draft the manuscript. All authors read and approved the final manuscript.

\section{Acknowledgements}

We are grateful to S. Derveaux of the CMGG (UGent) for his critical reading of the manuscript.

We acknowledge the support of the European Community of the FP6 (project: STREP EET-pipeline - Proposal N 037260). This study was further supported by the Industrial Research Fund of the University of Antwerp (UA-IOF), the Research Foundation - Flanders (FWO-G.0198.08) and the Institute for the Promotion of Innovation through Science and Technology in Flanders (IWT). It presents research results of the Belgian program of Interuniversity Poles of Attraction, initiated by the Belgian State, Prime Minister's Office, Science Policy Programming. KDP is post-doctoral researcher by the FWO. CK is supported by the IWT (N081373). DG is an UA-IOF fellow. 


\section{Author Details}

'Center for Medical Genetics, Ghent University Hospital, Ghent, Belgium, 2Applied Molecular Genomics Group, Department of Molecular Genetics, VIB, Belgium, ${ }^{3}$ University of Antwerp (UA), Antwerp, Belgium and ${ }^{4}$ Department of Pathology, Medical School of Valencia, University of Valencia, Valencia, Spain

Received: 12 October 2009 Accepted: 12 May 2010

Published: 12 May 2010

\section{References}

1. Costa JL, Meijer G, Ylstra B, Caldas C: Array comparative genomic hybridization copy number profiling: a new tool for translational research in solid malignancies. Semin Radiat Oncol 2008, 18(2):98-104

2. Shinawi $M$, Cheung SW: The array $\mathrm{CGH}$ and its clinical applications. Drug Discov Today 2008, 13(17-18):760-770.

3. Schouten JP, McElgunn CJ, Waaijer R, Zwijnenburg D, Diepvens F, Pals G: Relative quantification of 40 nucleic acid sequences by multiplex ligation-dependent probe amplification. Nucleic Acids Res 2002, 30(12):e57.

4. den Dunnen JT, White SJ: MLPA and MAPH: sensitive detection of deletions and duplications. Curr Protoc Hum Genet 2006, Chapter 7(Unit 7):14.

5. Multiplicon the multiplex experts [http://www.multiplicon.com]

6. Brouwers N, Sleegers K, Engelborghs S, Bogaerts V, Serneels S, Kamali K, Corsmit E, De Leenheir E, Martin JJ, De Deyn PP, et al:: Genetic risk and transcriptional variability of amyloid precursor protein in Alzheimer's disease. Brain 2006, 129(Pt 11):2984-2991.

7. Bogaerts V, Engelborghs S, Kumar-Singh S, Goossens D, Pickut B, Zee J van der, Sleegers K, Peeters K, Martin JJ, Del-Favero J, et al.: A novel locus for dementia with Lewy bodies: a clinically and genetically heterogeneous disorder. Brain 2007, 130(Pt 9):2277-2291.

8. Sleegers K, Brouwers N, Gijselinck I, Theuns J, Goossens D, Wauters J, DelFavero J, Cruts M, van Duijn CM, Van Broeckhoven C: APP duplication is sufficient to cause early onset Alzheimer's dementia with cerebral amyloid angiopathy. Brain 2006, 129(Pt 11):2977-2983.

9. Goossens D, Moens LN, Nelis E, Lenaerts AS, Glassee W, Kalbe A, Frey B, Kopal G, De Jonghe P, De Rijk P, et al: Simultaneous mutation and copy number variation (CNV) detection by multiplex PCR-based GS-FLX sequencing. Hum Mutat 2009, 30(3):472-476.

10. Brodeur GM: Neuroblastoma: biological insights into a clinical enigma. Nat Rev Cancer 2003, 3(3):203-216.

11. Van Roy N, De Preter K, Hoebeeck J, Van Maerken T, Pattyn F, Mestdagh P, Vermeulen J, Vandesompele J, Speleman F: The emerging molecular pathogenesis of neuroblastoma: implications for improved risk assessment and targeted therapy. Genome Med 2009, 1(7):74

12. Vandesompele J, Baudis M, De Preter K, Van Roy N, Ambros P, Bown N, Brinkschmidt C, Christiansen H, Combaret V, Lastowska M, et al: Unequivocal delineation of clinicogenetic subgroups and development of a new model for improved outcome prediction in neuroblastoma. J Clin Oncol 2005, 23(10):2280-2299.

13. Michels $E$, Vandesompele J, De Preter K, Hoebeeck J, Vermeulen J, Schramm A, Molenaar JJ, Menten B, Marques B, Stallings RL, Combaret V, Devalck C, De Paepe A, Versteeg R, Eggert A, Laureys G, Van Roy N, Speleman F: ArrayCGH-based classification of neuroblastoma into genomic subgroups. Genes Chromosomes Cancer 2007, 46(12):1098-108.

14. Tomioka N, Oba S, Ohira M, Misra A, Fridlyand J, Ishii S, Nakamura Y, Isogai E, Hirata T, Yoshida Y, et al:: Novel risk stratification of patients with neuroblastoma by genomic signature, which is independent of molecular signature. Oncogene 2008, 27(4):441-449.

15. Janoueix-Lerosey I, Schleiermacher G, Michels E, Mosseri V, Ribeiro A, Lequin D, Vermeulen J, Couturier J, Peuchmaur M, Valent A, et al:: Overall genomic pattern is a predictor of outcome in neuroblastoma. $J$ Clin Oncol 2009, 27(7):1026-1033.

16. Ambros PF, Ambros IM, Brodeur GM, Haber M, Khan J, Nakagawara A, Schleiermacher G, Speleman F, Spitz R, London WB, et al: International consensus for neuroblastoma molecular diagnostics: report from the International Neuroblastoma Risk Group (INRG) Biology Committee. $\mathrm{Br}$ J Cancer 2009, 100(9):1471-1482.

17. MRC-Holland (MLPA) [http://www.mrc-holland.com]

18. MRC Coffalyser MLPA-Dat [http://old.mlpa.com/coffalyser]

19. Villamón E, Piqueras M, Mackintosh C, Alonso J, de Alava E, Navarro S, Noguera R: Comparison of different techniques for the detection of genetic risk-identifying chromosomal gains and losses in neuroblastoma. Virchows Arch 2008, 453(1):47-55.

20. Arraycghbase [http://medgen.ugent.be/arrayCGHbase]

21. Menten B, Pattyn F, De Preter K, Robbrecht P, Michels E, Buysse K, Mortie G, De Paepe A, van Vooren S, Vermeesch J, et al: arrayCGHbase: an analysis platform for comparative genomic hybridization microarrays. BMC Bioinformatics 2005, 6:124

22. Olshen $A B$, Venkatraman ES, Lucito R, Wigler M: Circular binary segmentation for the analysis of array-based DNA copy number data. Biostatistics 2004, 5(4):557-572.

23. Bland JM, Altman DG: Measuring agreement in method comparison studies. Stat Methods Med Res 1999, 8(2):135-160

24. van Houte BP, Binsl TW, Hettling H, Pirovano W, Heringa J: CGHnormaliter: an iterative strategy to enhance normalization of array CGH data with imbalanced aberrations. BMC Genomics 2009, 10(1):401.

doi: $10.1186 / 1471-2164-11-298$

Cite this article as: Kumps et al., Multiplex Amplicon Quantification (MAQ), a fast and efficient method for the simultaneous detection of copy number alterations in neuroblastoma BMC Genomics 2010, 11:298

\section{Submit your next manuscript to BioMed Central and take full advantage of:}

- Convenient online submission

- Thorough peer review

- No space constraints or color figure charges

- Immediate publication on acceptance

- Inclusion in PubMed, CAS, Scopus and Google Scholar

- Research which is freely available for redistribution 